\title{
Time-Optimal Control Problem for a Fuzzy Object
}

https://doi.org/10.31713/MCIT.2020.01

\author{
Tatyana A. Komleva \\ Department of Mathematics \\ Odessa State Academy of Civil Engineering and Architecture \\ Odessa, Ukraine \\ Natalia V. Skripnik \\ Department of Optimal Control and Economic Cybernetics \\ Odessa I.I. Mechnikov National University \\ Odessa, Ukraine
}

\begin{abstract}
In this article one optimal control problem when the system behavior is described by linear fuzzy differential equations is considered. The conditions of its solvability are formulated, and the optimal time and optimal controls are obtained.
\end{abstract}

Keywords- fuzzy mapping; Hukuhara derivative; timeoptimal control; linear; differential equation

\section{INTRODUCTION}

Uncertainties in different scientific areas arise mainly from the lack of human knowledge. In many practical problems the uncertainties are not of statistical type. This situation occurs mainly in the case of modeling of the linguistic expressions appeared in different scientific areas because of dependence on human judgment. Also, the uncertainty in different measurements due to finite resolution and error of measuring instruments is in many cases more possibilistic than probabilistic, since, in many applications the measurements cannot be repeated.

The theory of fuzzy sets introduced by L.A. Zadeh [1], allow us to easily model these non-probabilistic uncertainties. This fact justifies the increasing interest in theoretical and practical aspects of fuzzy set theory lately. The applications of fuzzy set theory can be found in many branches of science such as physical, mathematical and engineering sciences [2-10]. Recently there have been new advances in the theory of fuzzy differential equations, fuzzy integro-differential equations, differential inclusions with fuzzy right-hand side and fuzzy differential inclusions as well as in the theory of control fuzzy differential equations, control fuzzy integro-differential equations, control fuzzy differential inclusions, and control fuzzy integro-differential inclusions (see [6-24] and references therein). This made it possible not only to consider new types of equations, but also to formulate new optimal control problems.

One of these problems - the time-optimal problem and the method for its solution are considered in this article.

\author{
Liliya I. Plotnikova \\ Department of Mathematics and Modeling Systems \\ Odessa National Polytechnic University \\ Odessa, Ukraine \\ Andrej V. Plotnikov \\ Department of Information Technology and Applied \\ Mathematics \\ Odessa State Academy of Civil Engineering and Architecture \\ Odessa, Ukraine \\ a-plotnikov@ukr.net
}

\section{PRELIMINARIES}

Let $R$ be the set of real numbers and $R^{n}$ be the $n$ dimensional Euclidean space $(n \geq 2)$. Denote by $\operatorname{conv}\left(R^{n}\right)$ the set of nonempty compact and convex subsets of $R^{n}$.

For two given sets $X, Y \in \operatorname{conv}\left(R^{n}\right)$ and $\lambda \in R$, the Minkowski sum and scalar multiple are defined by $X+Y=\{x+y \mid x \in X, y \in Y\}$ and $\lambda X=\{\lambda x \mid x \in X\}$.

Consider the Hausdorff distance $h(\cdot, \cdot)$ given by

$$
h(X, Y)=\min \left\{r \geq 0 \mid X \subset Y+B_{r}(0), Y \subset X+B_{r}(0)\right\},
$$

where $B_{r}(0)=\left\{x \in R^{n} \mid\|x\| \leq r\right\}$ is the closed ball with radius $r$ centered at the origin ( $\|x\|$ denotes the Euclidean norm).

It is known that $\left(\operatorname{conv}\left(R^{n}\right), h\right)$ is a complete metric space [8].

Let $E^{n}$ be a family of all $u: R^{n} \rightarrow[0,1]$ such that $u$ satisfies the following conditions:

1) $u$ is normal, i.e. there exists $x_{0} \in R^{n}$ such that $u\left(x_{0}\right)=1$;

2) $u$ is fuzzy convex, i.e. for any $x, y \in R^{n}$ and $0 \leq \lambda \leq 1$ $u(\lambda x+(1-\lambda) y) \geq \min \{u(x), u(y)\} ;$

3) $u$ is upper semicontinuous, i.e. for any $x_{0} \in R^{n}$ and $\varepsilon>0$ there exists $\delta\left(x_{0}, \varepsilon\right)>0$ such that $u(x)<u\left(x_{0}\right)+\varepsilon$ whenever $\left\|x-x_{0}\right\|<\delta\left(x_{0}, \varepsilon\right), x \in R^{n}$;

4) the closure of the set $\left\{x \in R^{n}: u(x)>0\right\}$ is compact.

If $u \in E^{n}$, then $u$ is called a fuzzy set, and $E^{n}$ is said to be a space of fuzzy sets. 


\section{Modeling, control and information technologies -2020}

Definition 1.[5] The set $\left\{x \in R^{n}: u(x) \geq \alpha\right\}$ is called the $\alpha$-level $[u]^{\alpha}$ of a fuzzy set $u \in E^{n}$ for $0<\alpha \leq 1$. The closure of the set $\left\{x \in R^{n}: u(x)>0\right\}$ is called the 0 -level $[u]^{0}$ of a fuzzy set $u \in E^{n}$.

Theorem 1. [5,25] (Stacking Theorem). If $u \in E^{n}$ then

1) $[u]^{\alpha} \in \operatorname{conv}\left(R^{n}\right)$ for all $\alpha \in[0,1]$;

2) $[u]^{\alpha_{2}} \subset[u]^{\alpha_{1}}$ for all $0 \leq \alpha_{1} \leq \alpha_{2} \leq 1$;

3 ) if $\left\{\alpha_{k}\right\}$ is a nondecreasing sequence converging to $\alpha>0$, then $[u]^{\alpha}=\bigcap_{k \geq 1}[u]^{\alpha_{k}}$.

Conversely, if $\left\{A_{\alpha}: \alpha \in[0,1]\right\}$ is the family of subsets of $R^{n}$ satisfying conditions 1) - 3) then there exists $u \in E^{n}$ such that $[u]^{\alpha}=A_{\alpha}$ for $0<\alpha \leq 1$ and $[u]^{0}=\overline{\bigcup_{0<\alpha \leq 1} A_{\alpha}} \subset A_{0}$.

Let $z \in R^{n}$ and $\theta(z)$ be the fuzzy set such that $[\theta(z)]^{0}=z$.

We define the sum $u+v$ and the scalar multiplication $\lambda u$ by $[u+v]^{\alpha}=[u]^{\alpha}+[v]^{\alpha}$ and $[\lambda u]^{\alpha}=\lambda[u]^{\alpha}$ respectively, for every $\alpha \in[0,1]$

Define $D: E^{n} \times E^{n} \rightarrow[0, \infty)$ by the relation

$$
D(u, v)=\sup _{0 \leq \alpha \leq 1} h\left([u]^{\alpha},[v]^{\alpha}\right)
$$

Then $D$ is a metric in $E^{n}$. Further we know that $[6,8]$ :

i) $\left(E^{n}, D\right)$ is a complete metric space,

ii) $D(u+w, v+w)=D(u, v)$ for all $u, v, w \in E^{n}$,

iii) $D(\lambda u, \lambda v)=|\lambda| D(u, v)$ for all $u, v \in E^{n}$ and $\lambda \in R$.

Definition 2.[6,8] A mapping $f:[0, T] \rightarrow E^{n}$ is called continuous at point $t_{0} \in[0, T]$ provided for any $\varepsilon>0$ there exists $\delta\left(\varepsilon, t_{0}\right)>0$ such that $D\left(f(t), f\left(t_{0}\right)\right)<\varepsilon$ whenever $\left|t-t_{0}\right|<\delta\left(\varepsilon, t_{0}\right), t \in[0, T]$. A mapping $f:[0, T] \rightarrow E^{n}$ is called continuous on $[0, T]$ if it is continuous at every point $t_{0} \in[0, T]$.

Definition 3.[6,8] A mapping $f:[0, T] \rightarrow E^{n}$ is called measurable on $[0, T]$ if for any $\alpha \in[0,1]$ the multivalued mapping $f_{\alpha}(t)=[f(t)]^{\alpha}$ is Lebesque measurable.

Definition 4. $[6,8]$ A mapping $f:[0, T] \rightarrow E^{n}$ is called integrably bounded on $[0, T]$ if there exists a Lebesque integrable function $k(t)$ such that $\|x\| \leq k(t)$ for all $x \in f_{0}(t), t \in[0, T]$.

Definition 5.[6,8] An element $g \in E^{n}$ is called an integral of $f:[0, T] \rightarrow E^{n}$ over $[0, T]$ if $[g]^{\alpha}=\int_{0}^{T} f_{\alpha}(t) d t$ for any $\alpha \in[0,1]$, where $\int_{0}^{T} f_{\alpha}(t) d t$ is the Hukuhara integral.

Theorem 2.[6,8] If a mapping $f:[0, T] \rightarrow E^{n}$ is measurable and integrably bounded then $f(t)$ is integrable over $[0, T]$.

Theorem 3.[6,8] Let $f, g:[0, T] \rightarrow E^{n}$ be integrable and $\lambda \in R$. Then

$\int_{0}^{T}(f(t)+g(t)) d t=\int_{0}^{T} f(t) d t+\int_{0}^{T} g(t) d t ; \int_{0}^{T} \lambda f(t) d t=\lambda \int_{0}^{T} f(t) d t$.

Let $A, B, C$ be in $\operatorname{conv}\left(R^{n}\right)$. The set $C$ is the Hukuhara difference of $A$ and $B$, if $B+C=A$, i.e. $C=A \frac{H}{} B$.

Definition 6.[6,8] A mapping $F:[0, T] \rightarrow \operatorname{conv}\left(R^{n}\right)$ is differentiable in the sense of Hukuhara at $t \in[0, T]$ if for some $h>0$ the Hukuhara differences $F(t+\Delta) \frac{H}{F(t),}$ $F(t) \frac{H}{H} F(t-\Delta)$ exist in $\operatorname{conv}\left(R^{n}\right)$ for all $0<\Delta<h$ and there exists $D_{H} F(t) \in \operatorname{conv}\left(R^{n}\right)$ such that

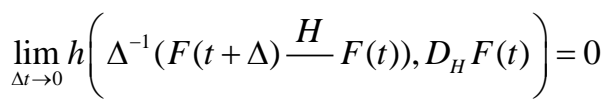

and

$$
\lim _{\Delta t \rightarrow 0} h\left(\Delta^{-1}\left(F(t) \frac{H}{H} F(t-\Delta)\right), D_{H} F(t)\right)=0 .
$$

Here $D_{H} F(t)$ is called the Hukuhara derivative of $F(\cdot)$ at $t$.

Definition 7.[6,8] A mapping $x:[0, T] \rightarrow E^{n}$ is called differentiable at $t \in[0, T]$ if for any $\alpha \in[0,1]$ the set-valued mapping $x_{\alpha}(t)=[x(t)]^{\alpha}$ is differentiable in the sense of Hukuhara at point $t$ with $D_{H} x_{\alpha}(t)$ and the family $\left\{D_{H} x_{\alpha}(t): \alpha \in[0,1]\right\}$ defines a fuzzy set $\dot{x}(t) \in E^{n}$.

If $x:[0, T] \rightarrow E^{n}$ is differentiable at $t \in[0, T]$, then we say that $\dot{x}(t)$ is the fuzzy derivative of $x(\cdot)$ at the point $t \in[0, T]$. 


\section{III.LINEAR CONTROL FUZZY PROBLEM}

Now, consider the control Cauchy problem

$$
\dot{x}(t)=v(t) x(t)+\theta(u(t)), \quad x(0)=x_{0},
$$

where $v:[0, T] \rightarrow[0,1]$ is a control, $\theta:[0, T] \rightarrow E^{n}$ is a fuzzy control such that $u \in R^{n}$ and $\left|u_{i}\right| \leq 1, i=\overline{1, n}, x_{0} \in E^{n}$.

The fuzzy mapping $x(\cdot, v, u)$ will be called the solution of the system (1) on the interval $[0, T]$ corresponding to the controls $v(\cdot)$ and $u(\cdot)$ if it is continuously and satisfies system (1) almost everywhere on $[0, T]$.

It is known [8], that the control problem (1) has a unique solution $x(\cdot, v, u)$ on the interval $[0, T]$ for all $v(\cdot), u(\cdot)$ and

$$
x(t, v, u)=e^{0} x_{0}^{t} v(s) d s \quad e^{\int_{0}^{t} v(s) d s} \int_{0}^{t} e^{-\int_{0}^{s} v(\tau) d \tau} \theta(u(s)) d s .
$$

We also note that any solution $x(\cdot, v, u)$ of the system (1) has the following property: for all $t \in[0, T]$, there always exist such a number $a(t, v, u) \geq 1$ and the vector $b(t, v, u) \in R^{n}$ that $[x(t, v, u)]^{\alpha}=a(t, v)\left[x_{0}\right]^{\alpha}+b(t, v, u)$ for all $\alpha \in[0,1]$.

Definition 8. The set $y \in E^{n}$ is homothetic to the set $x \in E^{n}$ if there exist a number $a>0$ and a vector $b \in R^{n}$ such that $[y]^{\alpha}=a[x]^{\alpha}+b$ for all $\alpha \in[0,1]$.

Consequently, the fuzzy set $x(t, v, u)$ is homothetic to the fuzzy set $x_{0}$.

Let $x_{K} \in E^{n}$. Suppose that fuzzy sets $x_{0}$ and $x_{K}$ are homothetic with parameters $a \geq 1$ and $b \in R^{n}$.

Consider the following time-optimal problem (Problem A.): it is required to move the object $x(\cdot, v, u)$ according to the system (1) from the initial set $x_{0}$ to the final set $x_{K}$ for the minimum time $T>0$, so that $x(T, v, u)=x_{K}$.

This problem is solvable in the class of constant controls.

Theorem 4. If fuzzy sets $x_{0}$ and $x_{K}$ are homothetic with coefficients $a \geq 1$ and $b \in R^{n}$ then the optimal control problem A can be solved and the optimal time $T^{*}$ and optimal controls $v^{*}$ and $u^{*}$ will be

$$
T^{*}= \begin{cases}b_{\max }, & a=1, \\ \ln (a), & a>1 \text { and } a-1 \geq b_{\max }, \\ \frac{\ln (a) b_{\max }}{a-1}, & a>1 \text { and } a-1<b_{\max },\end{cases}
$$

$$
v^{*}= \begin{cases}0, & a=1, \\ 1, & a>1 \text { and } a-1 \geq b_{\max }, \\ \frac{a-1}{b_{\max }}, & a>1 \text { and } a-1<b_{\max },\end{cases}
$$

$$
u_{i}^{*}= \begin{cases}\frac{b_{i}}{b_{\max }}, & a=1, \\ \frac{b_{i}}{a-1}, & a>1 \text { and } a-1 \geq b_{\max }, \\ \frac{b_{i}}{b_{\max }}, & a>1 \text { and } a-1<b_{\max },\end{cases}
$$

where $i=1, \ldots, n, \quad b_{\max }=\max _{i=1, n}\left|b_{i}\right|$.

Proof. Obviously, for some $T>0, v \in[0,1]$ and $u \in R^{n}$ the following equality

$$
e^{v T} x_{0}+e^{v T} \int_{0}^{T} e^{-v s} d s \theta(u)=x_{K}
$$

must hold.

As $\left[x_{K}\right]^{\alpha}=a\left[x_{0}\right]^{\alpha}+b$ for all $\alpha \in[0,1]$ then

$$
e^{v T}=a, \quad e^{v T} \int_{0}^{T} e^{-v s} d s \cdot u=b
$$

for all $\alpha \in[0,1]$.

Now we consider the following special cases.

Case 1. Let $a=1, b \in R^{n}$. Then $v^{*} \equiv 0$. Consequently, we have $T u=b$. Hence we have $T^{*}=b_{\max }$ and $u_{i}^{*} \equiv \frac{b_{i}}{b_{\max }}$, $i=\overline{1, n}$.

Case 2. Let $a>1, b \in R^{n}$. First we take $v \equiv 1$. By (3), we have

$$
e^{T}=a, \quad e^{T} \int_{0}^{T} e^{-s} d s \cdot u=b .
$$

From here we get $\quad e^{T}=a, \quad\left(e^{T}-1\right) u=b$.

If $a-1 \geq b$ then $T^{*}=\ln (a)$ and $u_{i}^{*} \equiv \frac{b_{i}}{a-1}, i=\overline{1, n}$.

Now we consider the case when $a-1<b$. Then we must take $0<v<1$ and there exists $j \in\{1, \ldots, n\}$ such that $\left|u_{j}\right| \equiv 1$ and $\left|b_{j}\right|=b_{\text {max }}$. By (3), we have

$$
e^{v T}=a, \quad e^{v T} \int_{0}^{T} e^{-v s} d s=b_{\max } .
$$

Consequently, $\quad T^{*}=\frac{\ln (a) b_{\max }}{a-1} \quad$ and $\quad v^{*}=\frac{a-1}{b_{\max }} \quad . \quad$ We substitute these values in (3) and get $u_{i}^{*} \equiv \frac{b_{i}}{b_{\max }}, i=\overline{1, n}$.

The theorem is proved. 


\section{Modeling, control and information technologies -2020}

Now we consider the case when the fuzzy set $x_{K}$ is not homothetic to the initial fuzzy set $x_{0}$. Obviously, the Problem A cannot be considered, since it will not have a solution. Therefore, further we will consider some other optimal control problems, but first we introduce some additional definitions.

Definition 9. A fuzzy set $y \in E^{n}$ is included in a fuzzy set $x \in E^{n}$ if $[y]^{\alpha} \subseteq[x]^{\alpha}$ for all $\alpha \in[0,1]$ and we write $y \subseteq x$.

Definition 10. A fuzzy set $y \in E^{n}$ has a nonempty intersection with a fuzzy set $x \in E^{n}$ if $[y]^{\alpha} \cap[x]^{\alpha} \neq \varnothing$ for all $\alpha \in[0,1]$ and we write $y \bigcap x \neq \varnothing$.

Now we consider some optimal control problems.

Problem B: it is required to move the object $x(\cdot, v, u)$ according to the system (1) from the initial set $x_{0}$ to the final set $x_{K}$ for the minimum time $T>0$ so that $x(T, v, u) \cap x_{K} \neq \varnothing$.

This problem always has a solution and this problem was considered in [24].

Problem C: it is required to move the object $x(\cdot, v, u)$ according to the system (1) from the initial set $x_{0}$ to the final set $x_{K}$ for the minimum time $T>0$ so that $x(T, v, u) \subseteq x_{K}$ and $D\left(x(T, v, u), x_{K}\right) \rightarrow \min$.

This problem does not always have a solution. It has a solution if there is a fuzzy set $y \in E^{n}$ that is homothetic to the initial fuzzy set $x_{0} \in E^{n}$ with coefficient $a \geq 1$ and it is contained in $x_{K} \in E^{n}$, i.e. $y=a x_{0}+b \subseteq x_{K}$. Obviously, this is not always possible. If it is possible, then we construct this fuzzy set $y \in E^{n}$ and then the problem is solved similarly to Problem A.

Problem D: it is required to move the object $x(\cdot, v, u)$ according to the system (1) from the initial set $x_{0}$ to the final set $x_{K}$ for the minimum time $T>0$ so that $x(T, v, u) \supseteq x_{K}$ and $D\left(x(T, v, u), x_{K}\right) \rightarrow \min$.

This problem does not always have a solution. It has a solution if there is a fuzzy set $y \in E^{n}$ that is homothetic to the initial fuzzy set $x_{0} \in E^{n}$ and it contains a fuzzy set $x_{K} \in E^{n}$, i.e. $y=a x_{0}+b \supseteq x_{K}$. Obviously, this is not always possible. If it is possible, then we construct this fuzzy set $y \in E^{n}$ and then the problem is solved similarly to Problem A.

\section{References}

[1] L.A. Zadeh, "Fuzzy sets," Inf. Control, no. 8, 1965, pp. 338-353.

[2] Gy. Bárdossy, J. Fodor, "Evaluation of uncertainties and risks in geology," Springer Verlag, Berlin Heidelberg, New York, 2004

[3] I.G. Perfil'eva, "Applications of the theory of fuzzy sets," J. Soviet Math., vol. 58, no. 2, 1992, pp. 148-194.

[4] H.-J. Zimmermann, "Fuzzy set theory and its applications," Springer Science + Business Media New York, 2001

[5] D. Dubois and H. Prade, "Fuzzy sets and systems," Academic Press, New York, 1980

[6] V. Lakshmikantham, and R.N. Mohapatra, "Theory of fuzzy differential equations and inclusions," Series in Mathematical Analysis and Applications, vol. 6. Taylor \& Francis, Ltd., London, 2003.

[7] S. Chakraverty, S. Tapaswini, and D. Behera, "Fuzzy Differential Equations and Applications for Engineers and Scientists," CRC Press, 2017.

[8] A.V. Plotnikov and N.V. Skripnik, "Differential equations with "clear" and fuzzy multivalued right-hand side. Asymptotics methods," AstroPrint, Odessa, 2009

[9] B. Bede, "Mathematics of fuzzy sets and fuzzy logic," Studies in Fuzziness and Soft Computing, vol. 295, Springer-Verlag, BerlinHeidelberg, 2013.

[10] B. Pękala, "Uncertainty data in interval-valued fuzzy set theory," Properties, Algorithms and Applications, Studies in Fuzziness and Soft Computing, vol. 367, Springer-Verlag, Berlin-Heidelberg, 2019.

[11] O. Kaleva, "Fuzzy differential equations," Fuzzy Sets Syst., vol. 24, no. 3, 1987, pp. 301-317.

[12] O. Kaleva, "The Cauchy problem for fuzzy differential equations," Fuzzy Sets Syst., vol. 35, no. 3, 1990, pp. 389-396.

[13] J.-P. Aubin, "Fuzzy differential inclusions," Probl. Control Inf. Theory, vol. 19, no. 1, 1990, pp. 55-67.

[14] V.A. Baidosov, "Fuzzy differential inclusions," J. Appl. Math. Mech., vol. 54, no. 1, 1990, pp. 8-13.

[15] T.E. Dabbous, "Adaptive control of nonlinear systems using fuzzy systems,” J. Ind. Manag. Optim., vol. 6, no. 4, 2010, pp. 861-880.

[16] E. Hullermeier, "An approach to modelling and simulation of uncertain dynamical systems," Internat. J. Uncertain., Fuzziness KnowledgeBased Systems, no. 7, 1997, pp. 117-137.

[17] T.A. Komleva, "The full averaging of linear fuzzy differential equations with $2 \pi$-periodic right-hand side," J. Adv. Res. Dyn. Control Systems, vol. 3, no. 1, 2011, pp. 12-25.

[18] N.A. Perestyuk, and N.V. Skripnik, "Averaging of fuzzy systems," Ukr. Math. J., vol. 70, no. 3, 2018, pp. 477-494.

[19] O. D. Kichmarenko and N.V. Skripnik, "The partial averaging of fuzzy differential equations with maxima," Adv. Dyn. Syst. Appl., vol. 6, no. 2, 2011, pp. 199-207.

[20] A.V. Plotnikov and N.V. Skripnik, "The generalized solutions of the fuzzy differential inclusions," Int. J. Pure Appl. Math., vol. 56, no. 2, 2009, pp. 165-172.

[21] A.V. Plotnikov, "The averaging of control linear fuzzy differential equations," J. Adv. Res. Appl. Math., vol. 3, no. 3, 2011, pp. 1-20.

[22] A.V. Plotnikov and T.A. Komleva, "Full averaging of control fuzzy integrodifferential inclusions with terminal criterion of quality," Int. J. Control Sci. Eng., vol. 3, no. 2, 2013, pp. 68-72.

[23] A.V. Plotnikov and T.A. Komleva, "The averaging of control linear fuzzy $2 \pi$-periodic differential equations," Dyn. Contin. Discrete Impuls. Syst., Ser. B. Appl. Algorithms, vol. 18, no. 6, 2011, pp. 833-847.

[24] A.V. Plotnikov, T.A. Komleva and A.V. Arsiry, "Necessary and Sufficient Optimality Conditions for a Control Fuzzy Linear Problem," Int. J. Industrial Mathematics, vol. 1, no. 3, 2009, pp. 197-207.

[25] M.L. Puri, and D.A. Ralescu, "Fuzzy random variables,” J. Math. Anal. Appl., no. 114, 1986, pp. 409-422. 